\title{
EFFECTIVENESS OF COMBINED USE OF ARM ABILITY TRAINING WITH ARM WEIGHT SUPPORT TO IMPROVE UPPER EXTREMITY FUNCTIONAL SKILLS AND ADL SKILLS IN PARKINSONISM PATIENTS
}

\author{
${ }^{1}$ Dr.Feba Jose, ${ }^{2}$ Dr. Surendra Kumar Meena, ${ }^{3}$ Dr. Neha Jain \\ ${ }^{I}$ Masters in occupational therapy (Adv. Neuroscience), \\ Mahatma Gandhi College of Occupational Therapy, Jaipur \\ ${ }^{2}$ Principal, Mahatma Gandhi Occupational Therapy College, Jaipur \\ ${ }^{3}$ Assot. Professor, Mahatma Gandhi college of Occupational Therapy College, Jaipur.
}

Article DOI: https://doi.org/10.36713/epra7786

DOI No: 10.36713/epra7786

\begin{abstract}
OBJECTIVE: This study is to determine the effectiveness of combined use of arm ability training with arm weight support to improve upper extremity functional skills and ADL skills in Parkinsonism patients.

METHOD: Thirty subjects with parkinsonism ( 5 months to 10 years of Parkinson's disease) were selected, who fulfilled the inclusion and exclusion criteria and according to the screening measures using UPDRS, Hoehn and Yahr scale and MMSE. Baseline assessment (Pre-test) was done using FMA, UEFI, and Katz index. After baseline assessment arm ability training (AAT) combined with arm weight support (45 minutes) was given to the patients for 12 weeks, 45 minutes in a day for 3 days in a week and post-intervention assessment (Post-test) was taken after 12 weeks by using the same outcome measures.

RESULT: Post-training there was a significant improvement in all the outcome measures. The result of the study showed evidence of benefit of the practice of Arm ability training with Arm weight support in Parkinsonism; enhance improvement as a result of Arm ability training along with Arm weight support were found in FMA-UE is $x=97.00$ (volitional movement), UEFI is $x=46.20$ ( Hand functionality), Katz Index is $x=5.20$ (Autonomy of ADL). The post-test has showed a greater improvement than from the pre-test in all the outcome measure (FMA-UE-P=0.001, UEFI-P=0.001 and Katz Index- P=0.001). The Pre and Post score comparison of outcome variables shown improvement in upper extremity functional skills and in Activities of Daily living skills following the intervention programme.

CONCLUSION: The Arm ability training techniques with Arm weight support was found to be effective in improving upper extremity functional skills as well as ADL skills among the Parkinsonism patients. The Arm ability training combined with Arm weight support can be used with other occupational therapy intervention to provide additional benefits to the Parkinsonism patients.
\end{abstract}

KEYWORDS: upper extremity functional skills, Activities of Daily Living, Arm ability training, Arm weight support, Parkinson's disease.

\section{SIGNIFICANCE OF THE STUDY}

This study is significant to endeavor in encouraging the upper extremity functional skill and ADL skill in Parkinson's patients. Hand function and finger agility are affected in Parkinsonism patients. These difficulties will affect the patient's daily life physically, mentally, leads to dependence in both activities of life (ADL) and quality of life (QOL). Generally, people with Parkinson's disease are referred to occupational therapy (OT) due to a remarkable physical disability, Occupational therapy aims to evaluate the patient's level of participation in ADL, to rule out the upper extremity functional difficulties that impede the autonomy of patients. Upper extremity-based occupational therapy intervention, planned as a role of a multidisciplinaryintegrated treatment, on upper extremity functional skills and enhancing independence in daily living in patients with PD. So this study especially aims to determine the effects of combined use of Arm ability 
training with Arm weight support for better upper extremity functions leads to an enhancement of independence in ADL skills in Parkinsonism.

\section{INTRODUCTION}

Parkinson's disease is also known as shaking palsy, it was described by Dr. James Parkinson in 1817. It is a chronic, progressive neurodegenerative disease, chiefly of a later stage of life that is linked with the disease in dopamine production in the substantia nigra and is marked by both motor symptoms, especially which include resting tremor, "cogwheel" rigidity, bradykinesia, and non-motor features like sleep disorder, depression, altered cognition. The most common cause of Parkinsonism is Parkinson's disease (PD). (Parkinson J.1817:1-16). Occupational therapy focuses on treating hand dysfunctions to decrease the dependency of patient's independence in ADL (C.E.Clarke, 1995) and need to be appraised as a prime feature of multidisciplinary technique. No former studies have conducted an inquiry into the efficacy of sensorimotor, upper extremity-based OT intervention, planned as a role of a multidisciplinary-integrated treatment, on upper extremity functional skills and enhancing independence in daily living in patients with PD. So this study is especially aimed to determine the effects of combined use of Arm ability training with Arm weight support for better upper extremity functions leads to an enhancement of independence in ADL skills in Parkinsonism.

\section{BACKGROUND}

The term Parkinsonism is a symptom elaborate to represent the motor features of Parkinson's disease which include resting tremor, bradykinesia, and muscular rigidity (Rajput AH, Rozdilsky, 1991). The common symptom of Parkinson's disease is Tremor. The motor symptoms of Parkinsonism are noted to the loss of striatal dopaminergic neurons, whereas the presence of non-motor symptoms is due to neural loss in the dopaminergic areas. Approximately $75 \%$ of patients with Parkinson disease (PD) has tremor during the illness; a rhythmical, involuntary oscillatory movement of the body is known as tremor (Twelves D, Perkins KS, 2003). Patient with a tremor has broad deficits in the performance components, including mobility, balance, hand dexterity, memory, executive functions (Shulman LM, 2008).As the course of the disease progression, the effectiveness of medication constantly decrease and daily functioning and social participation become increasingly impaired. Parkinson's disease (PD) has a marked impact on the quality of life of both the client and their caretaker. (Martignoni E, Citterio, 2011). The diagnostic features of PD are Bradykinesia (slowed movement), Hypokinesia (Poverty of movement), resting tremor, rigidity (Stanely Fahn, 2003). These features lead to impaired manual dexterity with progressive limitations in reaching, grasping, and diminished fine motor tasks have been traced in Parkinson's disease (PD). Up to the present date, the evidence-based intervention for upper limb strategies in parkinsonian patients is lacking. (Quinn L, Busse M, Dal Bello-Haas V, 2012).

The Arm Ability Training (AAT) (Platz T.2004, Deutsecher Wissenschafts-Verlag (DWV); 2006) (Impairment orient training) aims to train discrete abilities such as Aiming, speed, Dexterity, Tracing, and Steadiness. The Arm Ability Training (AAT) was developed to train all the sensorimotor abilities.

The application of Arm weight support (AWS) (weights) emerges from the advocacy in standard occupational therapy textbooks (Foti et al., 1996; Trombly, 1995; Turner et al., 1996). The modified tools used to enhance functional dexterity are focused on reducing tremors and to improving upper extremity function. Weighted utensils, weighted cups, weighted cuff, weighted wrist brace are used in decreasing tremors in PD. Few occupational therapists observe that using wrist weights, weighted cuffs and utensils are helpful for Parkinson's patients (Foti et al., 1996; Turner et al., 1996). Tasks like hammering and leather punching which needs more resisted grasps allow distal stability and decrease tremor and enhance upper extremity function which leads to improving the ADL skills in PD (Newman et al., 1995). Thus the Arm weighted support (AWS) has a major part in the treatment of PD. The use of weights in Parkinsonism where not been encouraged much in clinical practice.

The hand functions and finger agility are affected in PD. Such disruptions accompany dependence in activities of daily living (ADL) and poor quality of life (QOL) in Parkinsonism patients (Michele franciotta et al., 2019). These hand impairments will lead to (1) Decreased manual dexterity (2) loss of fine motor skills (3) difficulty in grip output (4) inability to carry out activities with normal amplitude, coordination, speed (Q. Whishaw et al., 2002 \& S. Pradhan et al., 2015). These skills are gained by motor learning activities that are associated with executive function (E.L. proud and M.E. Morris 2010\& C.Rodriguez-Aranda et al., 2016). Impaired hand function develops problems in activities of daily living (ADL), like eating, brushing, grooming, dressing, washing and writing (Y. Choi et al., 2017). And leads to deprivation of independence and reduces the QOL.

Occupational therapy focuses on treating hand dysfunctions to decrease the dependency of patient's independence in ADL (C.E.Clarke, 1995) and need to be appraised as a prime feature of multidisciplinary technique. No former studies have conducted an inquiry into the efficacy of sensorimotor, upper extremity-based OT intervention, planned as a role of 
a multidisciplinary-integrated treatment, on upper extremity functional skills and enhancing independence in daily living in patients with PD.

\section{Occupational Therapy Assessments}

1. Unified Parkinson's disease rating scale: This scale Assesses 4 sections

2. Mentation, behavior, and mood (4items), ADL (13 items), Motor examination (14 items), the complication of therapy (11 items)

3. All the sections except the 4 th component score use 5 point scale ranging from 0-4 (Fahn. S, 1987). In most of the obtainable $\mathrm{PD}$ rating scale, the UPDRS analyze the clinic metric effects of impairment and disability measurements for PD.

4. Hoehn and Yahr scale: The Hoehn and Yahr scale is a clinical severity rating scale solely confined to $\mathrm{PD}$ for categorizing motor functions, which defines the availability and limitations in motor function in PD (Melvin Yahr and Margaret Hoehn, 1967). It helps to identify the progression of the course of the disease with or without dopaminergic intervention.

5. Mini-mental state examination: The minimental state examination is a simple, short, standardized pen and paper test for the cognitive mental function in clients with a neurological condition. It is established to use quickly and easily admissible to the patient and for practitioners (Ingrid ArevaloRodriguez, 2015)

6. Fugl-Meyer assessment of upper extremity: It is a systemized assessment tool for poststroke recovery. The Fugl-Meyer assessment was evolved in 1975; upper extremity FuglMeyer (UEFM) is then determined, accepted stroke motor measures. FMA UE items are arranged into tools that observe the outlying movements that are progressively at the distal part of the upper extremity (Axel R. Fugl-Meyer; 1975). Assess 4 significant domains; motor capacity, sensation, passive joint development, joint pain.

7. Upper extremity functional Index: The upper extremity functional index (UEFI) is a self-reported questioner used as a measure to evaluate the functional deficits in the individual with abnormal musculoskeletal upper extremity functions (Stratford PW et al; 2001)

8. Katz index of independence in activities of daily living: Katz index of independence in activities of daily living is also known as Katz ADL it is the most relevant tool to assess the functional ability as a quantifying client's capacity to perform activities of daily living without any assistance (Katz S et al;1963).

\section{Occupational Therapy Intervention to Improve Upper Extremity Functional Skills and Activities of Daily Living Skills in Parkinsonism}

Arm Ability Training Tasks - The Arm Ability Training (AAT) aims to train discrete abilities such as Aiming, speed, Dexterity, Tracing, and Steadiness. The Arm Ability Training (AAT) was developed to train all the sensorimotor abilities. The preference of training the tasks covers the discrete sensorimotor abilities to dominate the resources and consequently broad in a period of application for motor performance in numerous contrasting circumstances that come across the daily life. Additionally, different struggle in task is executed in each kind of task training to increase motor learning.

Arm Weight Support - The application of Arm weight support (AWS) (weights) emerges from the advocacy in standard occupational therapy textbooks (Foti et al., 1996; Trombly, 1995; Turner et al., 1996). The modified tools used to enhance functional dexterity are focused on reducing tremors and to improving upper extremity function. Weighted utensils, weighted cups, weighted cuff, weighted wrist brace are used in decreasing tremors in PD. Few occupational therapists observe that using wrist weights, weighted cuffs and utensils are helpful for Parkinson's patients. Tasks like hammering and leather punching which needs more resisted grasps allow distal stability and decrease tremor and enhance upper extremity function which leads to improving the ADL skills in PD. Thus the Arm weighted support (AWS) has a major part in the treatment of PD.

\section{PROTOCOL}

Subjects were given arm ability training with arm weight support for 45 minutes ( 2 minutes break after every 10 minutes). Totally the intervention given for 12 weeks, 3 sessions per week.

\section{Protocol for Arm Ability Training with Arm Weight Support (45 Minutes)}

1. Arm weight support: The weighted wrist brace $(250 \mathrm{~g}-480 \mathrm{~g})$ was given to the patient and asked them to wear it along with Arm ability training tasks. In the first 2 months, only $250 \mathrm{~g}$ of weighted wrist brace was given as weighted support to the patient, in the 3rd month $480 \mathrm{~g}$ of weighted wrist brace was given during the Arm ability training tasks. As shown in figure 1 .

2. Arm ability training: The training consists of a total of 8 training tasks, each task is given for 5 minutes and after every two tasks, 2 
minutes break is given to the patients. The patients are made to sit in a chair with a table parallel to them in a comfortable position without shoulder elevated and depressed. The training tasks are as shown in figure 2, they contain:

a) Aiming (5 minutes): The patients are given a target (3-50mm distance) and a pointer/pen is given. Asked patient to hit the target with the pointer/pen as fast as possible with goaloriented hand movements. The task is repeated in both hands (10 repetitions in the first month, 20 repetitions in the second, 30 repetitions in the 3 rd month).

b) Tapping ( 5 minutes): The patients are asked to tap their thumb, index, middle finger on the table surface. The command is given to them to do in an alternative pattern and fast repetitive movements. The task is repeated in both hands. (10 repetitions in the first month, 20 repetitions in the second, 30 repetitions in the 3rd month).

c) Cancellation (5 minutes): Different sizes and shapes are printed on paper are given to the patients and asked to cancel a particular shape with the use of a pen. The command is given to the patient to stabilize their upper arm so that they can hold the pen in a precise finger movement. The task is repeated in both hands (10 repetitions in the first month, 20 repetitions in the second, and 30 repetitions in the 3rd month).

d) Turning (5 minutes): The coins (diameter 18$23 \mathrm{~mm}$ ) are arranged parallel to the patient on the table surface and asked them to turn the coins with finger and forearm supination and pronation. The task is repeated in both hands (10 repetitions in the first month, 20 repetitions in the second, 30 repetitions in the 3rd month).

e) Maze tracking (5 minutes): A printed maze tracking paper is given to the patient and asked them to track through the maze by using a pencil, which needs a slow, continuous, precise movement of the hand and visually guided movements. After every week different pattern of maze is given to the patients. The task is repeated in a dominant hand (10 repetitions in the first month, 20 repetitions in the second, 30 repetitions in the 3rd month).

f) Bolt and Nut (5 minutes): The patients are given Bolts (diameter 3,5,12mm) and Nuts and asked to screw the nuts and bolts. The command is given to change nuts and bolts to different sides (from right - left and left-right hand). These tasks facilitate good finger dexterity, aiming, steadiness. (10 repetitions in the first month, 20 repetitions in second, 30 repetitions in the 3 rd month).

g) Placing small objects (5 minutes): Ten small wooden blocks (Jenga blocks) are given to the patients and asked them to place the wooden blocks on top of each other. The command is given to use one hand at a time, by using proper finger dexterity, forearm pronation and supination, aiming, steadiness to complete the task. The task is repeated in both hands (5 repetitions in the first month, 10 repetitions in the second, 15 repetitions in the 3 rd month).

h) Placing large objects (5 minutes): A plastic jars with different weights and volumes are placed in front of the patients and asked them to place these jars from one side to another side (from right-left and left-right side) and also asked them to place the jars on top of each other jars. The task is repeated in both hands (5 repetitions in the first month, 10 repetitions in the second, 15 repetitions in the 3rd month).

\section{RESULT}

The raw scores of pre and post-test of 10 patients were subjected to the statistical analysis. Due to Covid 19 pandemic, the data collected is less than the number of participants mentioned in the guidelines. In this study 10 patients with Parkinsonism had a mean age of $74.3+/-9.79$ among them 4 of them are age between $60-70$ and 3 are between $70-80$ and 3 were above 80 years of age. 7 of them are males and 3 of them are females. Ten Parkinson's patients in the study has a mean UPDRS score of $14.60+/-5.44$ (nm- EDL) and 18.80+/- 6.19 (M-EDL), 35.80+/7.33 (Motor examination) and 9.70 +/- 2.26 (Motor complications). The percentage of Hoehn and Yahr score is $20 \%$ in stage 2, $50 \%$ in stage 3, and $30 \%$ in stage 4 of total $100 \%$. The study group had a mean MMSE score of 28.1+/- 1.101.

Baseline assessment was done using the FuglMeyer assessment of upper extremity, Upper extremity functional index, Katz activities of daily living. The 10 patients have a mean FMA pre-test score of 95.80 and standard deviation of 1.033, UEFI pre-test score of 41.80 and standard deviation of 3.994. The mean score of Katz's index pre-test is 3.60 and the standard deviation is .516. Recovery between baseline and post-intervention were evident on outcome variables as is apparent from mean summarized Tables, i.e. post-test mean score of FMA-UE is $x=97.00$, illustrated in (Table6), posttest mean score of UEFI is $x=46.20$, illustrated in (Table7) and the post-test mean score of Katz Index is $\mathrm{x}=5.20$ as shown in (Table 8). The post-test has shown a greater improvement than the pre-test in all the outcome measures (FMA-UE-P=0.001, UEFI$\mathrm{P}=0.001$, Katz Index- $\mathrm{P}=0.001)$. The Pre and Post 
score comparison of outcome variables shown improvement in upper extremity functional skills and Activities of Daily living skills following the intervention program.

\section{CONCLUSION}

This study evaluates the efficacy of a specified OT intervention, planned as a part of Multidisciplinary in-depth and combined rehabilitation therapy, on upper extremity functional performance, and autonomy in ADL in people with PD.

This chapter summarizes the finding and explains the present research based on the results. This study was conducted to find whether the Arm ability Training (AAT) combined with Arm weight Support (AWS) on upper extremity among Parkinson's disease is effective for improving the upper extremity functional performance and ADL skills. The idea of enhancing upper extremity functional skills and ADL skills through the use of Arm Ability Training combined with Arm weight support is important with potential implications for clinical practice. The occupational therapy intervention was given to different age group Parkinson's patients and it showed a significant effect on all age group patients.

As observed from the result, the number of male and female Parkinson's patients recruited in the study shows a significant improvement after occupational therapy intervention.

After comparing the pre-test and post-test score of Fugl- Meyer assessment of the Upper extremity with the help of screening tool used i.e. UPDRS, H\&Y, and MMSE, shows that the pretreatment mean score was lower than the posttreatment mean score and the difference between the pre and post mean score was statistically highly significant. So it indicates that OT intervention plays a major role in the improvement of upper extremity volitional movement.

Following the pre-test and post-test score of the Upper extremity functional index with the help of screening tool used i.e. UPDRS, H\&Y, and MMSE, shows that the pre-treatment mean score was lower than the post treatment mean score and the difference between the pre and post mean score was statistically highly significant. So it indicates that OT intervention plays a major role in the improvement in hand functionality.

Comparing the pre-test and post-test score of Katz index with the help of screening tool used i.e. UPDRS, H\&Y, and MMSE shows that the pretreatment mean score was lower than the posttreatment mean score and the difference between the pre and post mean score was statistically highly significant. So it indicates that OT intervention plays a major role in the improvement in the Autonomy in ADL.

The results of the study showed evidence of benefit of practicing Arm Ability Training combined with Arm weight support in Parkinson's disease; enhance improvement as a result of Arm Ability Training with Arm weight support were found in Fugl Meyer assessment for upper extremity (volitional movements), upper extremity functional index (hand functionality) and Katz index (Autonomy in ADL) in people with Parkinson's disease. The study demonstrates clear findings, those patients in early and in medium H\&Y stages gained results from occupational therapy treatment, as they have shown improvement in upper extremity movements, coordination, autonomy in daily activity skills.

Both the techniques combined to use a range of specifically designed discrete abilities, such as Aiming, Speed, Dexterity, Tracing and Steadiness ((Platz T.2004, Deutscher Wissenschafts-Verlag (DWV); 2006), which would have developed to train all the sensory-motor abilities; thereby enhancing ability to increase movements through a sensorymotor training on hand and upper extremities in PD (H\&Y stage 1-3) was effective (G. Taghizadeh, A. Azad, 2018).

The previous studies of Arm Ability training (Thomas Platz MD et al., 2018) developed to encourage manual dexterity rehabilitation for the post-stroke population with mild to moderate arm paresis has shown the outcome of AAT enhanced sensorimotor effects in ADL with long time effect. The training-induced improvement in upper extremity abilities is established on functional reorganization cortical-subcortical cerebral neural system with a partial organization and adaptation of ipsilateral ventral premotor cortex in stroke patients. So that motor learning can facilitate future executions of upper extremity skills. Thomas Platz developed the AAT technique to train the sensorimotor abilities and individual performance to promote motor learning and to increase the repetition of training tasks to find the variations in difficulty to perform the task.

The study by Irene H.L. Chan et al., 2016, conducted the study to examine the effect of arm weight support with Armeospring in sub-acute stroke with arm impairments. They found that Arm weight support training is useful for a patient with sub-acute stroke after 3 weeks of training. The outcome of their study has shown an active ROM in shoulder flexion in upper extremity score in Fugl-Meyer assessment (FMA). While the FMA hand component result had significant changes in gained scores of moderate and mild impairment.

Hewer et al (1972) pointed out the importance of using weights on hands with tremors. A total of 50 
participants with essential tremor, ataxia, multiple sclerosis, cerebellar deterioration, stroke, Parkinson's disease were selected for the investigation. The weights were manifested in the hands as a wrist strap/ belt. The tremor was monitored by using an accelerometer\& videotaping was done. The movements of the hands are observed and the severity of tremor has been recorded during drawing and writing. The outcome of their investigation tells that $36 \%$ of the participants have displayed an improvement in tremors while using weights in their hands during activity. These findings were similar to the recently published met- analysis which demonstrated that the use of Arm weight support had significant results in hand rehabilitation (Swetha Krishnaswamy et al, (2016).

Lee\& colleagues, 2011 outlined the enhancement in gross and fine motor performance of upper extremity in PD patients (H\&Y stage 2-3) with 4 weeks of training of constraint-induced movement therapy. In 2015 Mateos-To set and Colleagues illustrated development in manual dexterity and strength after a single 15-minute hand exercise session. The outcome of this study authenticates that a target-oriented, goal-based, motor cognitive training ${ }^{80}$ leads to improvement in upper extremity skills and ADL skills in PD.

The obtained data validate the effectiveness of Arm ability training with Arm weight support in upper extremity functional skills and ADL skills in $\mathrm{PD}$, and point out the benefit of occupational therapy intervention on upper extremity training and ADL training.

There is much need for this type of research in occupational therapy to see the more beneficial effects of Arm ability training with Arm weight support on upper extremity to improve hand function and ADL independence in Parkinson's disease. When Covid 19 pandemic is eradicated, will conduct the research on a community level and in rural population. Conduct a study by comparing both Arm ability training and Arm weight support on hand function and in ADL skills in PD.

\section{REFERENCES}

1. Parkinson J. Sherwood, Neely and Jones. An essay on the shaking palsy: London; 1817:1-16.

2. James Parkinson. An essay on the shaking palsy revised; Journal of neuropsychiatry clinical neurosciences. 2002;14(2)

3. Rajput AH, Rozdilsky B, Rajput A. Accuracy of clinical diagnosis in Parkinsonism; A prospective study. Canadian journal of neurology science. 1991; 18:275-278.

4. Twelves D, Perkins KS, Counsell C. Systemic review of incidence studies of Parkinson's disease; movement disorder. 2003;18:19-31

5. Shulman LM, Gruber-Baldini AL, Anderson KE, Vaughan CG, Reich SG, Fishman PS, Weiner WJ.
The evolution of disability in Parkinson disease, movement disorder society. 2008; 23:790-796.

6. Martignoni E, Citterio A, Zangaglia R, Godi L, Pacchetti C, Fundaro C, Corengia E, Bono $G$, Nappi G. How Parkinsonism influences life: the patients point of view; journal of neurology science. 2011; 32:125-131.

7. George DeMaagd, PharmD, BCPS; and Ashok Philip, PhD. Parkinson's Disease and Its Management, Part 1: Disease Entity, Risk Factors, Pathophysiology, Clinical Presentation, and Diagnosis, the Union University School of Pharmacy in Jackson, Tennessee.2015;40 (8).

8. Stanley Fahn. Description of Parkinson's disease as a clinical syndrome. The new York academy of sciences. 2003; 991:1-14.

9. Lori Quinn, Busse M, Dal Bello-Haas. Management of upper extremity dysfunction in people with Parkinson disease and Huntington disease: facilitating outcomes across the disease life span. Journal of hand therapy. 2012; 26(2):148-155.

10. Vand der Marck MA, Kalf JG, Sturkenboom IH, Nijkrake MJ, Munneke M, Bloem BR. Multidisciplinary care for patients with Parkinson's disease. Parkinson related disorder. 2009; suppl 3:s219-s223.

11. Ingrid HWM Sturkenboom, Maud J Graff, George $F$ Borm, Eddy MM Adang. Effectiveness of occupational therapy in Parkinson disease: study protocol for a randomized controlled trail. National library of medicine. 2013; 14:34. 2-8.

12. Dixon L, Duncan D, Johnson P, Kirkby L, O'Connell H, Taylor H, Deane KHO. Occupational therapy for patients with Parkinson's disease. Cochrane database systemic review. 2007; 3:CD002813.

13. Platz T, Winter T, Muller N, Pinkowski C, Eickhof C, Mauritz KH. Arm ability training for stroke and traumatic brain injury patients with mild arm paresis: A single-blind, randomized, controlled trial. Arch phys med rehabil. 2001; 82:961-968.

14. Thomas platz, Stefanie van kaick. Best conventional therapy versus modular impairment-oriented training for arm paresis after stroke: A singleblind, multicenter randomized controlled trial. Neurorehabilitation and neural repair, A sage journal. 2009; 23 (7):706-716.

15. G. Taghizadeh, A. Azad, S. Kashefi, S. Fallah, and $F$. Daneshjoo. The effect of sensory-motor training on hand and upper extremity sensory and motor functions in patients with idiopathic Parkinson disease. journal of hand therapy. 2018; 31(4) 486493.

16. K.S. Lee, W.H. Lee and S. Hwang. Modified constraint induced movement therapy improves fine and gross motor performance of the upper limb in Parkinson disease. American journal of physical medicine and Rehabilitation. 2011; 90(5) 380-386.

17. S. Mateos- Toset, I. Cabrera-Martos, I. TorresSanchez, A. Ortiz- Rubio, and M.C. Valenza. Effects of a single hand exercise session on manual dexterity and strength in person with Parkinson 
disease. A randomized control trail. PM\&R. 2016; 8(2) 115-122.

18. G. Maestri, R. Maestri, G. Bertotti et al., Intensive rehabilitation treatment in early Parkinson's disease: a randomized pilot study with a 2-year follow-up. Neurorehabilitation and Neural Repair. 2015; 29(2) 123-131.

19. G. Frazzitta, P. Balbi, R. Maestri, G. Bertotti, N. Boveri and G. Pezzoli. The beneficial role of intensive exercise on Parkinson disease progression. American journal of physical medicine \& Rehabilitation. 2013; 92(6) 523-532.

20. D.Ferrazzoli, P. Ortelli, R. Maestri et al., Does cognitive impairment affect rehabilitation outcome in Parkinson's disease?. Frontiers in Aging Neuroscience. 2016'8, 192. 